\section{Fragebogen zu TKI-Nebenwirkungen validiert}

\begin{abstract}
Hand-Fuß-Syndrom sowie Mukositis oder Stomatitis sind belastende Nebenwirkungen von Tyrosinkinasehemmern (TKI). Lebensqualitätsfragebögen für diese Toxizitäten fehlten bisher weitgehend. Nun wurde der HAMSIQ bei Patienten mit metastasiertem Nierenzellkarzinom und TKI-Therapie validiert.
\end{abstract}

$\mathrm{H}^{2}$ and-Fuß-Syndrom und Mukositiden oder Stomatitiden belasten die Patienten und können dadurch Compliance und Behandlungseffekt limitieren. Neben dem klinischen Effekt spielt daher die Lebensqualität unter der oft langfristigen TKI-Einnahme eine wichtige Rolle. Im Rahmen zweier kontrollierter Vergleichsstudien (COMPARZ und PISCES) mit TKI bei Patienten mit metastasiertem Nierenzellkarzinom (mRCC) entwickelten Onkologen auf Basis des Oral Mucositis Daily Questionnaire den HAMSIQ (Hand-Foot and Mucositis Se- verity and Impact Questionnaire). Er sollte die Standardfragebögen zur Lebensqualität um die spezifischen Besonderheiten von Hand-Fuß-Syndromen und Mukositis unter TKI bei Patienten mit mRCC ergänzen. Anhand der Daten der PISCES-Studie, in der Pazopanib mit Sunitinib verglichen wurde, validierten die Forscher den Fragebogen.

Eingesetzt wurde der HAMSIQ zu Behandlungsbeginn und dann alle 2 Wochen bis zum Ende der 10-wöchigen Studienphase. Dabei erwies sich der Fragebogen in der Studie als brauchbar: Alle
Items erfüllten die Standardanforderungen für interne Konsistenz. Zudem konnte der Fragebogen u. a. Patienten entsprechend ihres unterschiedlichen ECOGPerformancestatus erfassen und spiegelte klinische Veränderungen sowie Veränderungen der Schwere oraler $\mathrm{Ne}$ benwirkungen bzw. des Hand-Fuß-Syndroms im Behandlungsverlauf gut wider. Die Korrelation mit anderen Fragebögen war zufriedenstellend.

Fazit: Die Beeinträchtigung der Lebensqualität durch Hand-Fuß-Syndrom sowie Mukositis oder Stomatitis unter TKI wird mit dem neuentwickelten HAMSIQ erfasst. Barbara Kreutzkamp

Lai JS et al. Validation of a short questionnaire to measure symptoms and functional limitations associated with hand-foot syndrome and mucositis in patients with metastatic renal cell carcinoma. Cancer. 2016;122(2):287-95.

\section{Prophylaxe bei hochemetogener Chemotherapie}

\section{Chemotherapiebedingte Übelkeit und Erbrechen (CINV) gefährden den Erfolg onkologischer Therapien. Rolapitant ist ein neuartiger, langanhaltend aktiver Neurokinin-1(NK-1)-Rezeptorantagonist bei hochemetogener Chemotherapie.}

$\mathrm{B}$ is zu 120 Stunden nach Chemothera$\mathrm{B}_{\text {pie können CINV auftreten, deshalb }}$ benötigen Patienten einen mindestens 5 Tage währenden Schutz. Für eine randomisierte Studie wurden 554 Patienten gewonnen, die sich einer hochemetogenen Cisplatin-basierten Chemotherapie $\left(\geq 70 \mathrm{mg} / \mathrm{m}^{2}\right)$ unterzogen. Sie erhielten randomisiert entweder Rolapitant in einer Dosierung von 9, 22, 5, 90 oder 180 mg oder Placebo 2 Stunden vor Beginn des ersten Zyklus, außerdem Ondansetron und Dexamethason. Primärer Endpunkt war das komplette Ansprechen

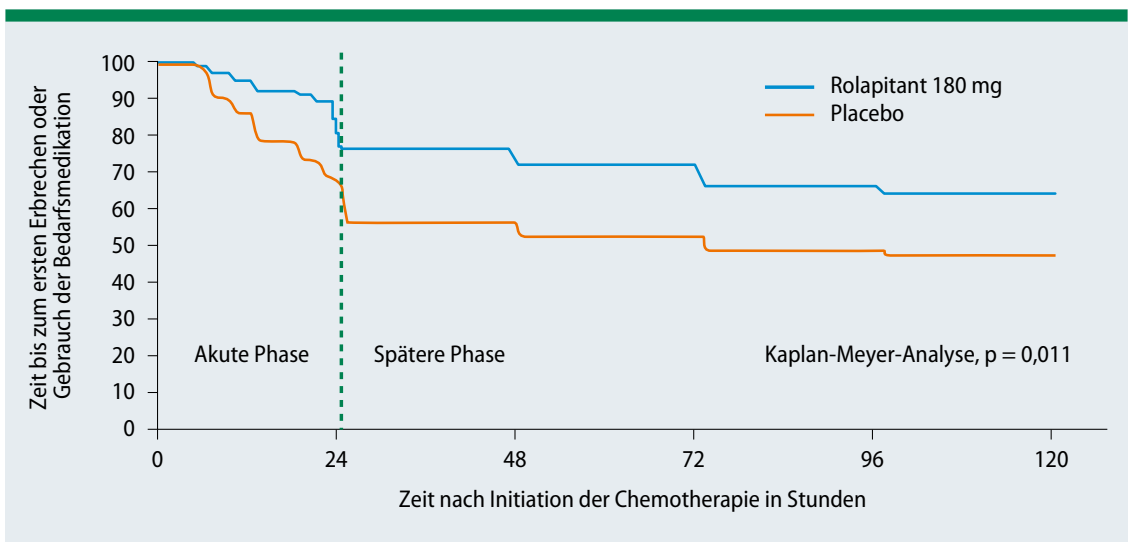

Abb. 1: In der Rolapitant-Gruppe war der Zeitraum bis zum ersten Erbrechen oder dem Gebrauch der Bedarfsmedikation im ersten Zyklus länger als in der Placebogruppe. (kein Erbrechen, kein Wechsel der Therapie) über 120 Stunden.

416 Patienten (91,6\%) führten den ersten Zyklus vollständig durch. Für alle Dosen war in allen Phasen das komplette Ansprechen besser als im Kontrollarm $(\mathrm{p}<0,05)$. Die größten Vorteile ergaben sich für $180 \mathrm{mg}$ gegenüber der aktiven Kontrolle über die gesamten 120 Stunden (62,5 vs. $46,7 \% ; \mathrm{p}=0,032)$, in der akuten $(87,6$ vs. $66,7 \%$; $p=0,001)$ und der verzögerten Phase (63,6 vs. $48,9 \% ; p=0,045)$. Im ersten Zyklus war die Zeit bis zum ersten Erbrechen oder dem Rückgriff auf die Bedarfsmedaktion in der Rolapitant-Gruppe länger als unter Placebo ( $p=0,011$; Abb. 1).

Fazit: Rolapitant war in allen Dosierungen gut verträglich und verbesserte das komplette Ansprechen gegenüber aktiver Kontrolle. $180 \mathrm{mg}$ waren zur Prophylaxe von CINV bei hochemetogener Chemotherapie grundsätzlich wie auch in der akuten und verzögerten Phase signifikant wirksam. Kathrin von Kieseritzky

Rapoport B et al. Study of rolapitant, a novel, long-acting, NK-1 receptor antagonist, for the prevention of chemotherapy-induced nausea and vomiting (CINV) due to highly emetogenic chemotherapy (HEC). Support Care Cancer. 2015;23(11):3281-8. 